Disclosure of Interests: Natalia Cid Boza: None declared, ML Velloso Feijoo: None declared, NAHIA PLAZA: None declared, Jose Luis Marenco Speakers bureau: abbie, pfizer, novartis, janmsen DOI: 10.1136/annrheumdis-2019-eular.5806

\section{AB0438 A STUDY COMPARING EFFICACY OF INTRA- ARTICULAR STEROID (IAS) VS INTRAARTICULAR SCLEROSANT IN PATIENTS WITH PERSISTENT SYNOVITIS OF KNEE IN RHEUMATOID ARTHRITIS}

Ramesh Ramamoorthy, Mythili Seethas, Balameena Kumar, Aravind Mani. Madras Medical College and Hospital, Rheumatology, Chennai, India

Background: Chronic persistent synovitis is commonly seen in inflammatory arthritis like RA where despite adequate DMARD therapy, few joints are chronically inflamed. They are the reason for increasing morbidity and poor functional status in these patients. Some patients show persistent synovitis despite intra-articular steroids and hence they is need to identify other drugs like sclerosant which can be of use in improving pain and functional status.

Objectives: To compare the efficacy of Intra-articular steroid versus sclerosant in rheumatoid arthritis (RA) with persistent synovitis despite optimum dose of csDMARDS and to determine, if sclerosant is superior/noninferior to steroids.

Methods: This is a single blinded-observational pilot study, conducted in Institute of Rheumatology, Madras Medical College for a period of 1 year. 20 patients with persistent synovitis (knees) despite optimum DMARD therapy are recruited as per inclusion and exclusion criteria. Disease and joint related activity and functional status are documented. Ethical committee approved the study. After getting written informed consent patients were randomized into two groups (A and B). Group A received IAS (Triamcinolone Acetonide) and group B received sclerosant (1\% Polidocanol). They are assessed at 1, 4, 12 and 24 week and various parameters documented. The results are analysed with SPSS v22 software.Inclusion Criteria: 1. RA diagnosis according to ACR/EULAR2010 criteria in the age between 20 and 70years with persistent synovitis of knee. 2.Patients not willing for biological therapy. Exclusion Criteria: 1. Age <20->70yrs. 2. Pregnant \& Lactating women. 3. Severe co-morbidities, clotting disorders and local infections. 4. Contraindications for intraarticular procedure. 5. Osteoarthritis and other arthritis and CTD. 6 Recent intra-articular injection within 3months.

Results: 40 patients were recruited for the study, with 20 in each group $45 \%$ patients in group $A$ and $65 \%$ patients in group $B$, showed significant improvement in DAS28, CRP, VAS pain and function. VAS scores improved within 1 week and no adverse effects were noted. Both the interventions found to be effective in reducing the pre operative VAS pain and function scores. However, Mean VAS Scores after 1 week of sclerosant injection found to be lesser than that of steroid group and the difference was statistically significant $(p<0.05)$.

Conclusion: Intra-articular sclerosant (1\%Polidocanol) is non-inferior to steroids in patients with persistent knee synovitis. It could be used as an effective alternative to steroids considering their side effect profile.

\section{REFERENCES}

[1] Use of objective measure of articular stiffness to record changes in finger joints after intra-articular injection of corticosteroid, annals of rheumatic diseases, 1997: 56:71-73

[2] Intra-articular injection with triamcilone hexacetonide in patients with rheumatoid arthritis: prospective assessment of goniometry and joint inflammation parameters. Rita Nely Vilar Furtado et al, Revista Brasileira de Reumatologia 2017:57(2):115-121

[3] Zulian F, Martini G, Gobber D, Agosto C, Gigante C, ZacchelloF. Comparison of intra-articular triamcinolone hexacetonide and triamcinolone acetonide in oligoarticular juvenile idiopathic arthritis. Rheumatology (Oxford). 2003; 42:1254-9.7.

[4] Hochberg MC, Chang RW, Dwosh I, Lindsey S, Pincus T, WolfeF. The American College of Rheumatology 1991 revised criteria for the classification of global functional status in rheumatoid arthritis. Arthritis Rheum. 1992; 35:498-502.9.

Disclosure of Interests: None declared

DOI: 10.1136/annrheumdis-2019-eular.5948

\section{AB0439 YTTRIUM-90 SYNOVIORTHESIS. OUR EXPERIENCE FOR} 25 YEARS

Patricia Lavilla ${ }^{1}$, M. Retuerto-Guerrero ${ }^{1}$, Sheila Melchor ${ }^{1}$, Álvaro García Martos ${ }^{2}$, Diana Vega ${ }^{3}$, Pilar Alonso ${ }^{1}$, Pilar F. Dapica ${ }^{1} .{ }^{1}$ Hospital universitario 12 de octubre, Rheumatology, Madrid, Spain; ${ }^{2}$ Hospital universitario del Tajo, Rheumatology, Madrid, Spain; ${ }^{3}$ Hospital universitario 12 de octubre, Radiology, Madrid, Spain

Background: Radioactive synoviorthesis (RS) is the intra-articular injection of a colloidal suspension of particles marked with a radioisotope that selectively irradiates the synovial membrane, respecting bone and cartilage. The radiocolloid is phagocytosed by type 2 synoviocytes of the synovial membrane, causing fibrosis and decreased production of synovia fluid. Intra-articular puncture is ensured by obtaining synovial fluid and then the radiopharmaceutical is instilled followed by $1 \mathrm{ml}$ of triamcinolone acetoide $(40 \mathrm{mg})$. After the procedure a graphic gamma image is made to assess the adequate distribution of the radionuclide in the joint cavity With a half-life of 2.5 days, the drug will continue to emit radiation for weeks, with symptomatic improvement which is observed from the second week. The main indication of this technique is refractory chronic synovitis to local and/or systemic treatment.

Objectives: To describe the clinical-demographic characteristics of patients treated with SR in our hospital, and to asses the efficacy and safety of this technique.

Methods: Retrospective observational study that analyze the radiosynoviorthesis practiced in the Nuclear Medicine service of the Doce de Octubre Hospital between January 1994 and December 2018 is analyzed. A tota of 113 techniques were analyzed in 89 patients from our center and other hospitals without Nuclear Medicine service, and data of 72 patients could be obtained from their clinical history. The efficacy of the technique was defined as total or partial, considering objective data (swelling and joint function) and subjective data (patient evaluation).

Results: 95 articular instillations were included in 72 patients, $46 \%$ women and $54 \%$ men; with an average age of $51.4 \pm 15$ |21-82| years, the knee articulations were injected with Yttrium-90 (5 millicuries). The patients had knee effusions lasting for an average of 18 months (IR 10 60). The temporal distribution was very heterogeneous, decreasing over the years (1994-1998: 23\%, 1999-2003: 32\%, 2004-2008: 20\%, 2009 2013: $16 \%, 2014-2018$ : $9 \%$ ). $93 \%$ of RS were indicated by the rheumatology service and $7 \%$ by traumatology. In the classification by patholo gies, $72.2 \%$ had systemic rheumatological disease. The most frequent causes of indication were rheumatoid arthritis (25\%), psoriatic arthropathy (24\%), spondyloarthropathy (17\%) and pigmented villonodular synovitis (11\%). Less frequent were juvenile idiopathic arthritis $(5 \%)$, arthrosis $(4 \%)$ microcrystalline arthritis (5\%) and non-specific chronic synovitis (9\%). $79 \%$ had been previously infiltrated with steroid, with an average of $2.8 \pm 1.8$ $|1-10|$ injections $/$ knee. The $79 \%$ had maintained treatment with NSAID the $42 \%$ with systemic steroid, the $55 \%$ with DMARDs and the $14 \%$ with biological therapy; appearing the therapeutic effect in the first 8 weeks in the $80 \%$ of the patients. 13 patients needed a reinstill with an average between radiosynoviorthesis of $25 \pm 21|6-80|$ months. Side effects were scarce $(2.8 \%)$ and local in nature; $8.3 \%$ required a knee prosthesis. Conclusion: RS has demonstrated acceptable efficacy and safety in cases of refractory synovitis of both mechanical and inflammatory etiology Despite this, it is a procedure little used nowadays.

Disclosure of Interests: None declared

DOI: 10.1136/annrheumdis-2019-eular.8168

\section{AB0440 CHANGES IN KEY LABORATORY VALUES WITH TOFACITINIB 5 MG BID TREATMENT IN PATIENTS WITH PSORIATIC ARTHRITIS AND RHEUMATOID ARTHRITIS}

William Rigby ${ }^{1}$, Gerd Rüdiger Burmester ${ }^{2}$, Oliver Fitzgerald ${ }^{3}$, Valderilio F. Azevedo ${ }^{4}$, Peter Nash ${ }^{5}$, Daniela Graham ${ }^{6}$, Cunshan Wang ${ }^{6}$, Thomas Jones ${ }^{7}$. ${ }^{1}$ Geisel School of Medicine at Dartmouth, Lebanon, NH, United States of America; ${ }^{2}$ Charité - University Medicine Berlin, Berlin, Germany; ${ }^{3}$ Department of Rheumatology, St Vincent's University Hospital, and Conway Institute for Biomolecular Research, University College Dublin, Dublin, Ireland; ${ }^{4}$ Universidade Federal do Paraná, Curitiba, Brazil; ${ }^{5}$ University of Queensland, Brisbane, Australia; ${ }^{6}$ Pfizer Inc, Groton, CT, United States of America; ${ }^{7}$ Pfizer Inc, Collegeville, PA, United States of America

Background: Tofacitinib is an oral Janus kinase inhibitor for the treatment of psoriatic arthritis (PsA) and rheumatoid arthritis (RA). In most countries where tofacitinib is approved, $5 \mathrm{mg}$ twice daily (BID) is the recommended dose for PsA and RA. An important component of any product labelling is information on the need for laboratory monitoring. 
Objectives: This post hoc analysis aimed to provide information on the effect of tofacitinib $5 \mathrm{mg}$ BID on laboratory values in PSA and RA patients (pts).

Methods: For analysis of pts with active PsA treated with tofacitinib 5 mg BID, data were pooled from 2 Phase 3 studies and an ongoing long-term extension (LTE) study (data cut-off, 25 January 2017; database not locked; data may change). For analysis of pts with moderate or severe RA treated with tofacitinib $5 \mathrm{mg}$ BID, data were pooled from 8 Phase 2, 7 Phase 3, and 1 LTE studies (data cut-off, 2 March 2017; for LTE, database not locked; data may change). All PsA and most RA pts received a background conventional synthetic disease-modifying antirheumatic drug. Data (to Month 12) for pts receiving constant tofacitinib $5 \mathrm{mg}$ BID were evaluated, comprising pts who received tofacitinib $5 \mathrm{mg}$ BID across studies, either at randomisation or following switch from placebo. Pts in the placebo groups who switched to tofacitinib $5 \mathrm{mg}$ BID at Month 3 were included from the time they first received tofacitinib. Pts who switched tofacitinib dose were excluded. Change from baseline in haematologic (haemoglobin, neutrophils, lymphocytes) and lipid (low-density lipoprotein cholesterol, high-density lipoprotein cholesterol, total cholesterol, triglyceride) levels and key liver tests (bilirubin, alanine aminotransferase, aspartate aminotransferase) were analysed. Although not addressed in the product labelling, creatine kinase, creatinine and C-reactive protein levels were also assessed. Pts meeting protocol-defined discontinuation criteria for laboratory values were evaluated.

Results: The constant tofacitinib $5 \mathrm{mg}$ BID group comprised 348 PsA pts and 3040 RA pts. Mean (standard error) changes/percentage changes from baseline for laboratory values are presented in the table. Laboratory values generally stabilised after 1 to 3 months, and lymphocyte levels stabilised by 24 months (data not shown). In both PsA and RA, $\leq 3.0 \%$ of patients met discontinuation criteria for any laboratory values.

Conclusion: In this post hoc analysis of laboratory data with tofacitinib 5 mg BID, changes in key laboratory values were similar for PsA and RA, and discontinuations due to protocol criteria being met for laboratory values were infrequent. These results provide further information on the effect of tofacitinib on laboratory values in PsA and RA.

Acknowledgement: This study was sponsored by Pfizer Inc. Medical writing support was provided by Karleen Nicholson, PhD, on behalf of CMC Connect, a division of McCann Health Medical Communications Ltd, Macclesfield, UK, and was funded by Pfizer Inc.

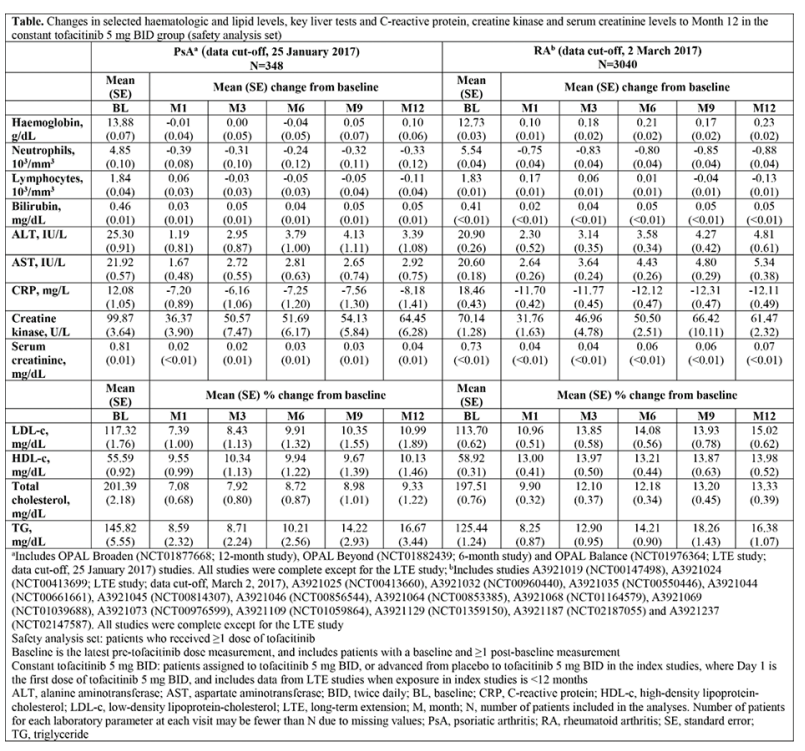

Figure 1

Disclosure of Interests: William Rigby Consultant for: Pfizer Inc, Gerd Rüdiger Burmester Consultant for: Roche, Sanofi-Genzyme, Speakers bureau: Roche, Sanofi-Genzyme, Oliver FitzGerald: None declared, Valderilio F Azevedo Grant/research support from: AbbVie, GSK, Janssen, Merck Serono, Novartis, Pfizer Inc, UCB, Consultant for: AbbVie, GSK, Janssen, Merck Serono, Novartis, Pfizer Inc, UCB, Peter Nash Grant/ research support from: AbbVie, Bristol-Myers Squibb, Eli Lilly, Janssen,
Novartis, Pfizer Inc, Roche, Sanofi, UCB, Consultant for: AbbVie, BristolMyers Squibb, Eli Lilly, Janssen, Novartis, Pfizer Inc, Roche, Sanofi, UCB, Speakers bureau: AbbVie, Bristol-Myers Squibb, Eli Lilly, Janssen, Novartis, Pfizer Inc, Roche, Sanofi, UCB, Daniela Graham Shareholder of: Pfizer Inc, Employee of: Pfizer Inc, Cunshan Wang Shareholder of: Pfizer Inc, Employee of: Pfizer Inc, Thomas Jones Shareholder of: Pfizer Inc, Employee of: Pfizer Inc

DOI: 10.1136/annrheumdis-2019-eular.1290

\section{AB0441 THERAPY WITH TOFACITINIB IN A COLOMBIAN POPULATION WITH RHEUMATOID ARTHRITIS: RESULTS OF THE DAILY CLINICAL PRACTICE}

Wilmer Gerardo Rojas Zuleta ${ }^{1}$, Oscar Jair Felipe Díaz ${ }^{1}$, Claudia Lucía Giraldo Herrera ${ }^{2}$, Adelis Enrique Pantoja Marquez ${ }^{1}$, Natalia Duque Zapata ${ }^{1}$, Monica Vasquez Jaramillo ${ }^{1}$, Catalina Orozco Gonzalez ${ }^{1}$, Jorge Hernando Donado Gómez.${ }^{1}$ Medicarte, Antioquia, Medellin, Colombia; ${ }^{2}$ Cali, Valle del Cauca, Cali, Colombia

Background: Rheumatoid arthritis (RA) is a chronic inflammatory disease that affects the quality of life and reduces life expectancy. It is characterized by the presence of autoantibodies and erosive synovitis that mainly involves small joints (1). Tofacitinib is the first oral Janus Kinase (JAK) inhibitor approved in 2012 for the treatment of patients with active, moderate to severe RA that does not respond to other therapies (2).

Objectives: The objective was to describe demographic and clinica results of a cohort of Colombian patients with RA treated with tofacitinib. Methods: Descriptive observational study of a historical cohort of RA patients in a specialized center for the management of inflammatory arthropathies, from April 2014 to February 2018. The following variables are described: age, sex, time evolution of the disease, type of AR, comorbidities, disease activity (DAS-28), schedule and treatment time. For the descriptive analysis, categorical variables are presented as absolute and relative frequencies; while continuous variables are presented as mean and standard deviation (SD) or median and interquartile range (IQR) according to distribution. The outcome of DAS $28 \leq 3.2$ was estimated by incidence rate, defined as the number of patients who presented the outcome divided by the summation of total exposure time of the patients.

Results: Of 59 patients included, $88.1 \%$ (52) were women, with a median age of 58.8 years (IQR 49-68 years), $74.6 \%$ were seropositive, the median time from diagnosis was 18.2 years (IQR 12- 28.3 years). High blood pressure was the most common comorbidity (40.7\%) and $7 \%$ had tuberculosis history. The median number of bDMARD prior to tofacitinib was 2 (IQR 1-3). Sixty six percent patients (39) were on monotherapy while $34 \%(20)$ were on combination with leflunomide (19) and methotrexate (1). The median time of treatment was 1.2 years (IQR $0.6-2$ years). At the beginning, $84.7 \%$ patients $(50)$ were in moderate or high disease activity and $15,3 \%$ (9) in remission or low activity; at the end of followup, $47.5 \%$ (28) were in remission or low activity and $52.5 \%$ (31) in moderate or high activity $(p=0.000)$. The mean DAS28 at the beginning of tofacitinib was $4.6 \pm 1.55$ and at the end of the follow-up was $3.5 \pm$ 1.49 , with a difference of means of 1.10 (IC95\% 0.62-1.57), (p 0.00). During the follow-up period, the rate of development of remission or low activity was $11.92(95 \% \mathrm{Cl} 8.35-16.51)$ cases per 100 people-month observed ( $p$ 0.00). Only 2 patients developed therapeutic failure.

Conclusion: Tofacitinib shows a good profile of effectivity in patients with failure to prior bDMARD. Almost $50 \%$ patient reaches low disease activity or remission during follow up and low number of therapeutic failure was found.

\section{REFERENCES}

[1] Picchianti-Diamanti A, et al. Front Microbiol. 2018;8(2696):1-9

[2] Cohen S, et al. Rheumatol Ther. 2018;5(1):283-291

Disclosure of Interests: Wilmer Gerardo Rojas Zuleta Speakers bureau: Bristol-Myers Squibb, Novartis, Jannsen, Pfizer, Oscar Jair Felipe Díaz Speakers bureau: Bristol-Myers Squibb, Novartis, Jannsen, Pfizer, Claudia Lucía Giraldo Herrera Speakers bureau: Novartis, Abbvie, Adelis Enrique Pantoja Marquez: None declared, Natalia Duque Zapata: None declared, Monica Vasquez Jaramillo: None declared, Catalina Orozco Gonzalez: None declared, Jorge Hernando Donado Gómez: None declared DOI: 10.1136/annrheumdis-2019-eular.5552 